Recepción: 22/ 04/ 2017

Aceptación: 26 / 05/ 2017

Publicación: 01/ 09/2017
Ciencias de la educación

Artículo de investigación

\title{
Importancia de la investigación etnográfica en el ejercicio profesional del trabajo social
}

Importance of ethnographic research in the professional practice of social work Importância da pesquisa etnográfica na prática profissional do trabalho social

Wadson Pinchi-Ramírez ${ }^{1}$

wprunt@hotmail.com

Correspondencia:wprunt@hotmail.com

I Doctor en Sociología, Maestro en Ciencias Sociales mención en Antropología Social, Docente de la Universidad Nacional de Trujillo, Perú. 


\section{Resumen}

Este trabajo expone aspectos preliminares de los momentos que la investigación se va incorporando en la utilización metódica en trabajo social. Para ello se abordarán tres momentos: 1. Se analizan los inicios de la etnografía tradicional y la práctica del trabajo social en el contexto de las instituciones públicas y privadas, centrándose en el accionar concreto y operativo, a través del estudio de casos de tratamiento directo e indirecto.

2. Se reflexiona sobre la investigación etnográfica en el ejercicio profesional del trabajo social y se desarrolla básicamente para entender los procesos comunitarios en el ámbito de la intervención profesional.

3. Se plantean los procesos socioculturales complejos emergentes, así como la etnografía científica y los retos del trabajo social donde se constata la construcción social de nuevos escenarios al igual que, la emergencia de actores sociales y culturales diversos, que en este marco se redefine el quehacer del ejercicio del trabajo social.

La investigación etnográfica descriptiva se ve limitada por estos nuevos cambios, por consiguiente, aparece la necesidad de replantear la investigación etnográfica, para crear las bases sólidas de la investigación/intervención profesional del trabajo social.

Palabras clave: etnografía; investigación; intervención; trabajo social.

\section{Abstract}

This work exposes preliminary aspects of the moments that the investigation is incorporated into the methodical use in social work. For this, three moments will be addressed:

1. The beginnings of traditional ethnography and the practice of social work in the context of public and private institutions are analyzed, focusing on concrete and operational actions, through the study of cases of direct and indirect treatment.

2. Ethnographic research is reflected on the professional practice of social work and is basically developed to understand community processes in the field of professional intervention.

3. Emerging complex sociocultural processes are considered, as well as scientific ethnography and the challenges of social work where the social construction of new scenarios is verified, as well as the emergence of diverse social and cultural actors, which in this context redefines the task of the exercise of social work. 
Descriptive ethnographic research is limited by these new changes, therefore the need to rethink ethnographic research appears, to create the solid bases of the research / professional intervention of social work.

Keywords: ethnography; investigation; intervention; social work.

\section{Resumo}

Este trabalho expõe aspectos preliminares dos momentos em que a investigação é incorporada no uso metódico no trabalho social. Para isso, três momentos serão abordados:

1. São analisados os inícios da etnografia tradicional e a prática do trabalho social no contexto das instituições públicas e privadas, com foco em ações concretas e operacionais, através do estudo de casos de tratamento direto e indireto.

2. A pesquisa etnográfica se reflete na prática profissional do trabalho social e é basicamente desenvolvida para compreender os processos comunitários no campo da intervenção profissional.

3. Considera-se processos socioculturais complexos emergentes, bem como a etnografia científica e os desafios do trabalho social onde se verifica a construção social de novos cenários, bem como o surgimento de diversos atores sociais e culturais, que neste contexto redefine a tarefa do exercício do trabalho social.

A pesquisa etnográfica descritiva é limitada por essas novas mudanças, portanto, a necessidade de repensar a pesquisa etnográfica aparece, para criar bases sólidas da pesquisa / intervenção profissional do trabalho social.

Palavras chave: etnografia; investigação; intervenção; trabalho social.

\section{Introducción}

La construcción del campo de acción y la redefinición del quehacer del trabajo social, especialmente en el Perú, ha recorrido o transitado diferentes contextos sociopolíticos, culturales e institucionales. En consecuencia está marcado por los diferentes cambios que se han experimentado en las sociedades y culturas latinoamericanas del siglo XX.

Ciertamente, el transcurrir del trabajo social en el Perú también se ve influenciado por los cambios producidos en los enfoques teóricos y metodológicos de las ciencias sociales, y la necesidad de incorporar los aportes de otras disciplinas. Como afirma Zamanillo, Teresa (2008), ciertamente el devenir del trabajo social, su nacimiento y posterior desarrollo está marcado por 
una profunda renovación en las ciencias sociales. Sin embargo, el quehacer cotidiano de la disciplina como profesión, no le ha permitido incorporar los aportes de los nuevos campos epistemológicos. Se centró básicamente en la intervención de los problemas sociales, principalmente en la práctica, así como la urgencia por la resolución de los problemas, a solucionar casos concretos del individuo y la familia.

En este sentido, hoy día, los enfoques teóricos metodológicos en la formación científica del trabajo social, argumentan la necesidad de una formación sistémica de la articulación entre investigación, intervención y transformación. Siendo el eje del nuevo debate la necesidad de construir la teoría y el método en el trabajo social.

Se pretende exponer aspectos preliminares de los momentos donde la investigación se va incorporando en la utilización metódica en trabajo social y el nacimiento de la perspectiva etnográfica, para que el crecimiento científico del trabajo social sea un factor gravitante de la intervención profesional integral en los contextos locales, globales, emergentes al igual que espacios individuales, grupales y comunitarios.

\section{Desarrollo}

La etapa inicial de la tradición etnográfica fue muy débil, centrándose en los estudios e intervención profesional en el ámbito urbano de servicios de salud y del mundo laboral de instituciones públicas y privadas (en el caso peruano a partir de la década treinta del siglo XX).

Ésta, se manifiesta a través del estudio de casos con una intervención que consiste en un tratamiento directo o indirecto, teniendo como base la descripción de los contextos sociales del individuo y la familia.

Se considera que "La meta de la etnografía es coger el punto de vista del nativo, su relación con la vida, darse cuenta de la visión del mundo" (Malinowski, citado por Spradley James, 1980b, p. 3), de igual modo, ella, tiene por trabajo describir una cultura donde su objetivo central es comprender otra forma de vista.

Para ello el procedimiento específico es el trabajo de campo, el cual involucra el estudio disciplinado de lo que es el mundo para la gente el cual ha aprendido a ver, oír, hablar, pensar y actuar en forma diferente. Más que estudiar a la gente, la etnografía significa aprender de la gente 
(Spradley James 1980b), entonces el quehacer del trabajo social ha estado limitado en el uso del método etnográfico para la interpretación y comprensión cultural en la intervención social de los casos.

En un segundo momento la investigación etnográfica en el ejercicio profesional del trabajo social, acentúa los mecanismos de la investigación-acción como una forma de conocer la realidad social y desenvolver el proceso de intervención social. Aparece el análisis de la realidad basado en los diagnósticos y la identificación de necesidades, en un ámbito socio comunitario (Rubio, Ma. José y Jesús Varas; 1999).

De manera general la tradición etnográfica tiene su sustento en los aportes de la antropología cultural desde la perspectiva norteamericana y aún más recientemente desde los aportes etnográficos de los estudios en los países latinoamericanos. Esta tradición se desarrolló básicamente para entender los procesos comunitarios en el ámbito de la intervención profesional. En este marco los estudios culturales, a través de las investigaciones preliminares y los diagnósticos específicos facilitaron la descripción e interpretación del universo simbólico de las prácticas colectivas en comunidades rurales, específicamente en el Perú para entender así el mundo andino (cultura Quechua y Aymara) y su relación con el mundo occidental. Estas prácticas se centraron básicamente, a partir de la construcción social simbólica, la conducta de los individuos y de los grupos sociales.

Actualmente, la investigación etnográfica cualitativa incorpora elementos e indicadores que ayudan a direccionar la necesidad de conocer y aplicar los estudios etnográficos, no solo para la interpretación del comportamiento colectivo comunitario sino también para la identificación de casos individuales, familiares y grupales; de igual manera, para la comprensión integral de los fenómenos y hechos culturales en el mundo de sus vidas cotidianas.

El primer paso metódico e instrumental del trabajo de campo etnográfico es la observación participante (Spradley, James 1980a), seguido de la entrevista etnográfica (Spradley 1980 b), ambos recorren tres momentos como:

- Inicio de la investigación.

- Recopilación de los datos. 
- Análisis de lo que se encuentra (interpretación-explicación) - elaboración del reporte.

Pero una condición básica del investigador es saber y comprender que la gente en cualquier lugar tiene un modo de vida, una cultura propia y si nosotros queremos comprender la naturaleza humana debemos comprender ello seriamente (Spradley James, 1980ª p.2).

Tomando como eje la tradición cultural de los aportes etnográficos, el ejercicio del trabajo social en el ámbito profesional, produce tendencias y estrategias específicas para la comprensión de la construcción social simbólica en un contexto de diversidad social y cultural. La pluralidad cultural, étnica y social se expresa a través de realidades complejas, que muchas veces dificulta el conocimiento objetivo solo desde una perspectiva cuantitativa.

En este sentido la necesidad de desarrollar descripciones, interpretaciones y explicaciones etnográficas es de suma importancia para argumentar adecuadamente las nuevas tendencias metodológicas de la re-conceptualización en la intervención del trabajo social para un nuevo tipo de promoción y desarrollo comunitario, que incorpore los procesos interculturales de comunicación y de articulación social de los procesos de ciudadanización urbana popular y rural (Pinchi, Wadson 2001), en las sociedades y movimientos sociales emergentes en espacios locales y globales.

Las construcciones actuales de los contextos sociales y culturales brindan como abordaje de estudio e investigación, diversas puertas de entrada a partir de las tensiones y las complejidades que los configuran. En este sentido "la realidad se construye a través de imágenes, sean éstas observadas tanto iconográficas o bien proyectadas por vías del habla, así se va formando una trama que urde con palabras una densa y expansiva matriz de relaciones fenoménicohermenéuticas que, a nivel de la conciencia y del lenguaje arman un tejido gramatical y semántico capaz de concatenar un corpus de vivencias, a través de las cuales se dejan traslucir y se transparentan nuestras más diversas experiencias" (Yáñez, Víctor; 2013:13).

En los escenarios actuales es posible reconocer mecanismos de comprensión que se expresan a través de conceptos dicotómicos como exclusión/integración, discriminación/no discriminación, marginación étnico-racional/inclusión sociocultural, inequidad/equidad. Frente a estos contextos la preocupación de la transformación social se ve desafiada por el encuentro cotidiano en estas complejidades, "lo que ha conducido a diversas reacciones dentro de las cuales aparece el 
desencanto en torno a los alcances de la disciplina en su campo de acción, se reniega de la complejidad de lo real actuando solo desde tradiciones fijas y aceptadas, sin considerar que se tiene al alcance posibilidades de revelarse en contra de posturas estáticas que no aportan a los procesos de desnaturalización de esta condición. La disciplina de trabajo social ha podido optar por ubicarse en la tensión entre lo justo e injusto provocando desde aquí un movimiento en torno a la generación de procesos de intervención que den cuenta de las complejidades existentes en el abordaje de la construcción de los fenómenos sociales contemporáneos (Hernández, Natalia; p.74).

En el Perú se configuran diversos procesos socioculturales complejos donde emergen diferentes actores sociales, andinos, amazónicos, afroperuanos, asiáticos de fuerte tradición cultural, así como europeos que reflejan sus manifestaciones culturales y sociales, que definen el nuevo escenario del "mestizaje" y "criollismo" nacional en diversos escenarios espaciales y sociales ya sean urbanos o rurales. Las migraciones verticales y horizontales completan el proceso de reconfiguración social, cultural, ambiental y político institucional que constituyen el nuevo cuadro del desborde estatal de fuerte contenido popular (Matos Mar José, 2012), que traen consigo nuevos retos académicos y científicos para describir y explicar estos procesos. Interpretar y comprender son los componentes actuales de la investigación etnográfica científica para crear nuevas bases de la teoría y el método que ayuden a redefinir el objeto de intervención del ejercicio profesional del trabajo social.

La tradición etnográfica de la simple descripción se vuelve insuficiente para comprender la visión y el mundo de vida cotidiana desde lo individual, grupal y comunitario, de lo andino y lo amazónico en la sociedad urbana y rural existentes.

Al decir de Aguayo, Cecilia (2014), nos interesa dar cuenta de la categoría "mundos de vida de los migrantes" como una acción profesional del trabajador social. "los profesionales del mundo social se insertan en el corazón de los puntos de vista de las comunidades y de los ciudadanos, es donde hacemos el quehacer profesional funcional, normativo e institucionalizado; pero también es desde donde se puede desplegar un horizonte de posibilidades" (Aguayo, Cecilia 2014 p. 2627), de esta manera "potenciar los valores aprendidos por los sujetos en los mundos de vida como soportes simbólicos y narrativos con que debe contar una argumentación valórica, donde 
responda a los contextos y sus exigencias" (Roig, 2002: 223 citado por Aguayo, Cecilia; 2014. p. 27).

Aguayo Cecilia (2014), la acción social intercultural, y por tanto la acción (ejercicio) profesional del trabajo social, requieren incorporar categorías más articuladoras en el contexto local, nacional y global. El conocimiento de la acción humana requiere del esfuerzo de la interpretación donde la tradición, la creación y la memoria son soportes básicos en los cuales se encuentra la identidad cultural de los pueblos latinoamericanos.

Hoy se constata fuertes brechas sociales, desigualdades e inequidades ciudadanas. La percepción de la construcción ciudadana en particular de los migrantes andinos y amazónico en términos imaginarios o ideales (derechos civiles y políticos) así como reales y prácticos solo se evidencia en la dimensión de supervivencia bajo nivel de conocimientos de derechos reconocidos por el Estado (Pinchi, Wadson 2005).

La cultura ciudadana es aún débil, existe marginalidad y exclusión. El proceso de construcción ciudadana, tiene que ser parte de contexto de intervención del trabajo social. Percibir estas dimensiones culturales desde los propios actores sociales es una necesidad, que invita, a volver a mirar desde nuevas aristas etnográficas. Entrar desde la visión del investigado en una relación sujeto-sujeto que exige nuevos entrenamientos en investigación etnográfica, solo así transcenderá la disciplina científica del trabajo social.

¿Cómo entender ahora los procesos subjetivos e intersubjetivos, tanto del sujeto investigador y del sujeto investigado?, por ejemplo, las emociones. La etnografía tradicional no lo pudo abordar desde lo cultural, lo dejó a un enfoque individualista desde el yo individual, desde la psicología, considerando que la emoción se ratifica en el lado individual de la contradicción individuo/sociedad, al margen de las relaciones sociales.

Actualmente la concepción tradicionalista de la etnografía es muy limitada y superficial al decir de Rosana Guber (2001), "esta concepción incidió profundamente en la metodología de la investigación suprimiendo las emociones del investigador, pero también las de los informantes, sin permitir encarar a la emoción como un fenómeno sociocultural con distintas expresiones y fundamentos" (p.109). 
Los estudios e investigaciones realizadas desde el trabajo social hasta la actualidad, coinciden que "es conveniente continuar con los procesos de investigación en torno a la práctica cotidiana del ejercicio profesional del trabajador social, con la intencionalidad de profundizar en los hallazgos planteados y de esa manera desarrollar elementos que aporten a la interpretación y comprensión desde las voces de los actores sociales (sujetos sociales-trabajadores sociales), como (sic) viven y sienten al igual que como aprecian las tendencias. Es urgente consolidar líneas de investigación según problemas sociales y la línea de investigación y trabajo social" (Falla, Uva; 2014. P. 134).

Es importante entender en el debate de la investigación etnográfica científica contemporánea las consecuencias prácticas que se producen a partir de la investigación y la intervención en una realidad concreta. Comprometen a una reflexión profunda en torno a preguntas como: ¿por qué y para qué se investiga?; para quién y con quién se investiga? "Las respuestas conllevan decisiones a partir de condiciones reales a partir de los objetivos que se pueden proponer, ¿hasta qué punto tiene sentido comprometerse en procesos para una investigación sin saber bien a donde vamos y cuáles son algunas de las condiciones básicas de las cuales partir ihasta dónde nos podemos involucrar? (Cifuentes, Rosa; 2011. p. 45).

Las respuestas exigen una profunda y permanente reflexión en la búsqueda de conocimientos de realidades sociales y culturales complejas, simbólicamente complementarias de condiciones y naturaleza humana plurales, de esta manera la investigación etnográfica tiene que trascender el campo de investigación realizada en una experiencia particular, para articularse en investigaciones etnográficas interdisciplinarias que den respuesta a los procesos complejos de construcción social de la realidad.

En consecuencia, es importante que la investigación etnográfica tome en cuenta que, "la característica más llamativa de la actual producción teórica de las ciencias sociales es la discusión y cuestionamiento de los diversos modos de acercarse a la realidad" (Nugent, Guillermo; 2016. p. 17).

La permanente crítica y reflexión de la forma de abordar la realidad social debe ser el camino permanente de la investigación etnográfica científica, para de esta manera trascender el único modo de encarar el conocimiento en las ciencias sociales, que considera la realidad social como 
algo "objetivo", en el sentido de hechos externos y cognoscibles, únicamente desde el punto de vista del observador. Esta única forma de proceder con un criterio, de racionalidad instrumental implicaría que una gama muy amplia de problemas quedaría relegados a lugares de poca importancia para ser abordados en la investigación al igual que en el campo de las ciencias sociales y del trabajo social en particular. Porque este proceder estaría evidenciado las serias limitaciones para describir e interpretar sistémicamente las culturas y las relaciones de interdependencia de procesos intersubjetivos en escenarios complejos de interculturalidad.

\section{Referencias Bibliográficas}

Aguayo, Cecilia (2014). La investigación ética moral con migrantes en salud primaria en Chile: desafíos interculturales. En: Ruth Izana Ibaceta (compiladora) 2014. Trabajo social e investigación. Buenos Aires: Espacio editorial

Cifuentes Gil, Rosa (2011). Diseño de proyectos de investigación cualitativa. Buenos Aires: Noveduc

Falla, Uva (2014). La investigación en el trabajo social contemporáneo. Colombia Universidad Colegio Mayor de Cundinamarca

Guber, Rosana (2001). La Etnografía. Método, campo y reflexividad. Bogotá -Colombia: Grupo Editorial Norma

Hernández, Natalia (2014). Investigación en contextos contemporáneos del trabajo social, una clave para las transformaciones complejas. En: Ruth Izana Ibaceta (compiladora) 2014. Trabajo social e investigación. Buenos Aires: Espacio editorial

Matos Mar, José (2012). Perú Estado desbordado y sociedad nacional Emergente. Historia corta del Proceso peruano: 1940-2010. Lima: Universidad Ricardo Palma/Editorial Universitaria

Nugent, Guillermo (2016). Errados y errantes. Modos de comunicación en la cultura peruana. Lima: La siniestra ensayos, Estación la cultura

Pinchi, Wadson (2005). Migración Andina, y cultura y construcción de la ciudadanía en el nuevo espacio periférico de Trujillo. En revista ciencia y tecnología, $\mathrm{N}^{\circ} 1,2005$. Trujillo-Perú: Universidad nacional de Trujillo, Escuela de Postgrado 
Pinchi, Wadson (2001). Construcción cultural de la ciudadanía urbana de los migrantes en Trujillo. Tesis para optar el grado de doctor en sociología. Lima-Perú: Universidad Inca Garcilaso de la Vega, Escuela de postgrado

Rubio, Ma. José y Jesús y varas (1999). El análisis de la realidad en la intervención social. Métodos y técnicas de investigación. Segunda edición. Editorial CCS. Madrid-España

Sprandley, James P. (1980a). La observación participante. Traducido del inglés por Rosa Zavala de Cilloníz. (1996). Nueva York: Editorial Holt Rinehart and Winston

Sprandley, James P (1980b). La entrevista Etnográfica. Traducido del inglés por Rosa Zavala de Cillóníz (1997). Nueva York: Editorial Holt Rinehart and Winston

Yáñez, Víctor (2013). El trabajo social en contextos de alta complejidad. Reflexiones sobre el Pensum Disciplinar. Buenos Aires: Espacio editorial

Zamanillo Teresa, (2008). Trabajo social con grupos y pedagogía ciudadana. Madrid: Editorial Síntesis S.A. 